\title{
Paint thickness control method Based on the film accumulation model
}

\author{
L. Yu, Y.Y. Zhang \\ China Aviation Planning and Construction Development Co. Ltd, Beijing 100120, China
}

\begin{abstract}
Since parameters of the traditional paint thickness accumulation rate model is difficult to determine, the model has issues as requiring a lot of repeat painting and so on. This paper presents a model of paint spray control model based on the film accumulation model to analyze how different trajectory curve affect the shape of film, the gaffe situation of thickness and width of paint film around the corner and at the gradient change place, and constructs accumulation model that holding both film thickness and width at a uniform lev-el at different curve. The accumulation model have different spray position requirements based on the trajectory curves to solve the paint film uniformity problems at three-dimensional trajectory space and improve the quality of the painting. Simulation results show that the proposed paint thickness control model can solve the thickness and width uniformity issues in a better way.
\end{abstract}

KEYWORD: Film accumulation model; Oval $\beta$ model; Cumulative rate; Thickness control

\section{INTRODUCTION}

Robots used to complete the work of surface coating or spray painting are productions of robot technology and surface paint technique, which is a special type of industrial robots (LIU Quanyu, 2015). The main advantage of using painting robots are making automated paint operations possible, which avoid that artificial painting workers get acute or chronic poisoned in a toxic environment, improve product quality and stability, reduce paint and energy consumption and improve labor productivity (ZHAO Hongjian, 2013). At present, research of the painting process parameter optimization are mostly focused on the following areas: the impact of spraying parameters toward color, thickness uniformity of the film and paint utilization. However, taking several quality requirements optimizing problems into account are not very common (HE Fujun, 2013). General Optimization do research about how to take best use of "limited" resources or find out the best allocation.

Hyotyiemi et al did research on the screen painting model and trajectory design optimization problem, and presented Gaussian model (WANG Chaojun, 2015) and the Cauchy distribution model (Ming Ying, 2015). Suhs et al (Suhs, 2015) proposed the concept of a limited range models, they assume that the thickness of spray film is uniform in a circular spray area. Based on the spray painting model, they have developed offline programming system has a parameter setting, solid modeling, simulation analysis and features as automatically generating the trajectory,. (Wang Yi et al) came up with one kind of cone-type paint film thickness model theory, which can obtain the effective average film thickness equation through the mathematical derivation. (Zhang, Yonggui) from Xi'an University of Technology proposed oval dual distribution model, which is a function of the film thickness distribution that is closer to the actual fog tapered distribution function. (Xia Wei et al) came up with part triangular mesh and paint deposition model, which provide a new method to divide the surface. The main parameters of dividing the surface will be determined by film thickness model, which make the system be able to handle paint intelligent trajectory planning of complex topologies part surface. ( $\mathrm{Yu}$ Shengrui et al) derive the geometric plane modeling problem into free-form surface modeling problem, which laid a solid foundation for calculating the free surface film thickness.

\section{CLASSIC PAINTING THICKNESS ACCUMULATION RATE MODEL}

In the actual production of air spray, in order to make the film get uniform accumulation in the painted surface, we will use compressed air to squeeze conical mist sprayed paint at the spout in the center of the gun nozzle. So that paint coverage 
area on the surface of the painting will be oval. The wheel shape of film thickness distribution hub at different cross-section on the same axis of the are similar. The further the distance away from the center, $\mathrm{t}$ smaller the cross-sectional ordinate values of the film thickness. So taking a cross-section into account, it can be described using $\beta$ distribution. Assume that the film thickness of the cross section at both $x$ and $y$ direction fit distribution that has the same $\beta$ value, the thickness of the cross-section along the lateral direction $x$ and longitudinal direction $y$ of the spray elliptical area fits $\beta$ distribution as well. Then, at direction $x$ where $y=k$, the film accumulation thickness model can be presented as following:

$$
d_{y-k}=d_{\max , y k}\left[1-\frac{x^{2}}{a^{2}\left(1-\frac{y^{2}}{b^{2}}\right)}\right]^{\hat{\alpha}-1}-a \sqrt{1-\frac{y^{2}}{b^{2}}} \leq x \leq a \sqrt{1-\frac{y^{2}}{b^{2}}}
$$

at direction $y$ where $x=1$, the film accumulation thickness model can be presented as following:

$$
d_{x-l}=d_{\max , x l}\left[1-\frac{y^{2}}{a^{2}\left(1-\frac{x^{2}}{b^{2}}\right)}\right]^{\beta_{2}-1}-b \sqrt{1-\frac{x^{2}}{a^{2}}} \leq y \leq b \sqrt{1-\frac{x^{2}}{a^{2}}}
$$

In both equation, $d_{\text {max }, y k}$ is the maximum number of the thickness of film.

The maximum $d$ of different $y$ direction crosssection appears near axial $x$, while the maximum $d$ of different $x$ direction cross-section appears near axial $y$. So that $d_{\text {max } y k}$ and $d_{\text {max } x l}$ represent $d_{\max }\left(1-\frac{y^{2}}{b^{2}}\right)^{\beta_{2}-1}$ and $d_{\max }\left(1-\frac{x^{2}}{a^{2}}\right)^{\beta_{1}-1}$, substitute which into equation (1) and (2), we can get

$$
\begin{aligned}
& d(x, y)=d_{\max }\left(1-\frac{y^{2}}{b^{2}}\right)^{\beta_{2}-1}\left[1-\frac{x^{2}}{a^{2}\left(1-\frac{y^{2}}{b^{2}}\right)}\right]^{\beta_{1}-1} \\
& \left(-b \leq y \leq b \quad-a \sqrt{1-\frac{y^{2}}{b^{2}}} \leq x \leq a \sqrt{1-\frac{y^{2}}{b^{2}}}\right)
\end{aligned}
$$

Oval dual $\beta$ distribution model is better than normal distribution model when it is used to describe the film accumulation situation, which meets the actual situation of the painting situation, however in the real painting process, the boundaries can not be as clear as the ideal state, therefore, a lot of parameters such as $a, b, \beta_{1}$ is difficult to determine. Then, multiple sets of data fitting analysis will be needed and the decomposition calculation will be a bit tedious.

\section{THE BUILD OF ROBOTIC SPRAY ACCUMULATION MODEL}

The accumulation rate of the known film plane is: $G=f(r, h), G$ is the thickness of the film that is $r$ from the center point on the plane. we control the distance between the gun and the working surface and make sure the distance $h$ remains unchanged, then:

$$
\begin{aligned}
& G=f(r, h)=A\left(R^{2}-r^{2}\right) \\
& \begin{cases}G=f(r, h) & (-R \leq r \leq R) \\
G=0 & (|r| \geq R)\end{cases}
\end{aligned}
$$

Here $A$ is the constant term, $R$ is radius of the working surface are for film spray.

After the film accumulation rate $G$ is known, we will do integration to time, we can get the coating thickness of a point on a plane during motion situation.

$q(r)=2 \int_{r}^{R} \int_{0}^{T} f(r, h) d r d t$

$T$ is the time the gluing process is needed when the nearest distance to the central spray point is $r$,

$T=\frac{2 \sqrt{R^{2}-r^{2}}}{v}$

$v$ is the linearly moving speed of the robot hand, when the height is $h, R$ is the maximum radius of the spray. To sum up, film thickness of the point that is $r$ away from the track center in the plane is as following:

$q(r)=2 \int_{r}^{R} \int_{0}^{\frac{2 \sqrt{R^{2}-r^{2}}}{v}} f(r, h) d r d t$

When there is uneven film thickness problem caused by a gradient difference, according to film accumulation rate model: $G=f(r, h)$, film thickness is related to the distance between the gun and the working surface. As far as we make sure the distance $h$ between the gun and the working surface are parallel to the normal vector of centerline and working surface, it will work out. When the gun arrives in radians of gradient difference $\theta_{2}$, we can adjust the angle of the end effector arm gesture $\theta_{2}$ and adjust the distance between the gun and the working surface according to the gradient difference $\Delta h$ between surfaces as Figure 2 shows and keep the curve normal vector parallel with the gun.

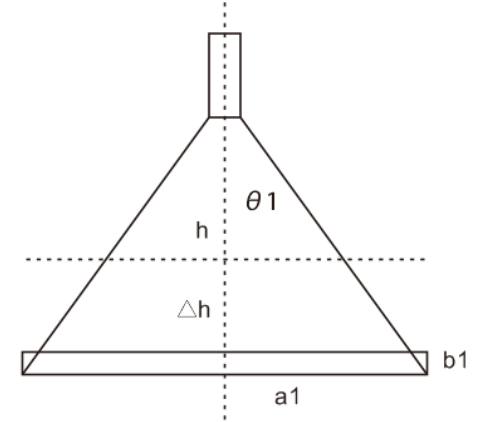

Fig2 Gradient difference $\Delta h$

At the corner, the changing of film thickness are caused by the changing of the spray area when the gun is moving across. According to the relationship 
between the distance $h$ from the spray gun to the working surface and the cumulative rate, we can adjust the normal vector of the distance between the gun and the working surface and the gun to control the film morphology.

Triangular $\triangle A B C$ is the film cross-section while doing normal straight line spraying. Here, $B C=a, A O=h$, the angle is $\theta_{1}$. We can see from the formula derived above that $S_{1}<S<S_{2}$. In the corner region, film will get thicker, outside the region will become smaller. We are inspired by the previous film variation rules to incline the spray gun to an angle $\theta_{2}$ and make sure $B C=D N=A, F H=h$. Because the inclination angle of the center line of the gun is $\theta_{2}$, thus $\angle H D E=\theta_{2}$. In order to get the length of $D E$, we will make a auxiliary line $M N \perp M E$. According to the apparent meaning of the questions, we know that:

$$
\begin{aligned}
& \angle M E C=\theta_{1} \Rightarrow D E=\frac{a}{\cos \theta_{2}-\sin \theta_{2} \cdot \tan \theta_{1}} \\
& F G=h_{1}=\left(\cos \theta_{2}-\sin \theta_{2} \tan \theta_{1}\right) \times h
\end{aligned}
$$

From the above equation, the adjustment of $\theta_{2}$ can draw the corresponding need to adjust the distance $\Delta h$ between the spray outlet to the working surface. According to the film accumulation model:

$$
q(r)=2 \int_{r}^{R} \int_{0}^{\frac{2 \sqrt{R^{2}-r^{2}}}{v}} f(r, h) d r d t
$$

we can see that the film thickness at a point is associated with the position $r, h$, as well as the robot hand moving speed. According to Goodman's technical measurements, we can get the accumulation rate data measured at different locations of the point list taken on the plane, then, we can get functional relationship between $r$ and $G$ :

$G=f(r, h)=A\left(R^{2}-r^{2}\right)$

$q(r)=2 \int_{r}^{R} \int_{0}^{1} f(r, h) d \rho d t$

Making the gun mouth as the origin, we can establish a Cartesian coordinate system. It is known:

$O D=h_{1}=\left(\cos \theta_{2}-\sin \theta_{2} \cdot \tan \theta_{1}\right) \times h ; A B=a$ that are the requirements of glue gun width and film height under linear case. In order to find out the film thickness of any point on the plane, we set the distance between point $p$ and spray track center as $r$, it is that the coordinates of point $p$ in the direction $R x$ is $r$, then:

$h_{y}=O D+D C+C M$

$=\left(1+\tan \theta_{1} \tan \theta_{2}\right) \times\left(\cos \theta_{2}-\sin \theta_{2} \times \tan \theta_{1}\right) \times h+|r| \times \tan \theta_{2}$

Under the situation that vertical plane have curved track and the radius of curvature is $R$. The glue discharged will be as follows if we take the distance of the respective arcs as we do it in a straight line:

$$
q(r) \times d r \times \frac{n \pi R}{180}
$$

After we take the arcs into consideration, the glue accumulation amount will be

$$
\begin{aligned}
& q_{1}(r) \times d r \times \frac{n \pi(R-r)}{180} \\
& q_{1}(r)=\frac{R}{R-r} q(r)
\end{aligned}
$$

From the above, we know if we want to have the same thickness level as in the straight line at the same point $r$, it will have to satisfy the following equation:

$$
\begin{aligned}
& q_{1}(r)=\frac{R}{R-r} q(r)=q\left(r_{x}, h_{y}\right) \\
& h_{y}=\left(1+\tan \theta_{1} \tan \theta_{2}\right) \times\left(\cos \theta_{2}-\sin \theta_{2} \times \tan \theta_{1}\right) \times h \\
& +r \times \tan \theta_{2} \\
& q\left(r_{x}, h_{y}\right)=4 A\left[\frac{2}{3}\left(h_{y} \cdot \tan \theta_{1}\right)^{3}-\left(h_{y} \cdot \tan \theta_{1}\right)^{2} r+\frac{1}{3} r^{3}\right] \\
& \times \frac{\sqrt{\left(h_{y} \cdot \tan \theta_{1}\right)^{2}-r^{2}}}{v}
\end{aligned}
$$

\section{EXPERIMENTAL RESULT ANALYSIS}

Based on the previous film accumulation model we use Matlab to do model simulation tests to the spray model. We will compare spray-sectional situation through simulation before and after optimization. Simulation steps are as follows:

Step 1: We will determine the initial conditions of the simulation model, which include setting spray width $30 \mathrm{~mm}$, that is, the bottom radius of the gun surface is $R=15 \mathrm{~mm}$, gun spread angle is $45^{\circ}$. According to the request, the distance between spray gun and the working surface when painting in a straight line is $36 \mathrm{~mm}$. By testing data that spraying targeting certain points on a plane spraying, we obtained cumulative model coefficients $A=1 / 8$.

Step Two: Then, we can write Matlab procedures, according to the accumulation mathematical model that established on characteristics of the track of small curvature and places have linear gradient difference, combing with the spray technology, we turn the mathematical model into Matlab statements controlled model, using Matlab to do the model simulation.

The third step: We will do simulation to places respectively have constant height, gradient difference and curvature of the corner without adjustment, as well as curvature corners that have made adjustment to the spray gun. 

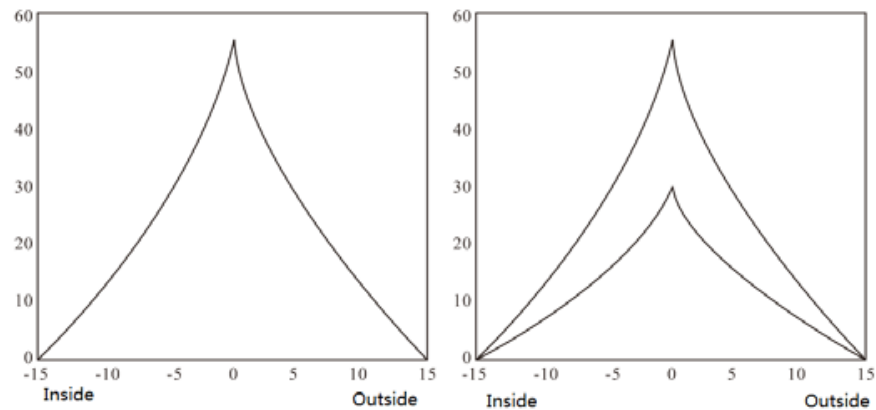

a. Straight line

b. Gradient difference is $10 \mathrm{~mm}$

Fig3 There is film gradient in the form (unit: $\mathrm{mm}$ )
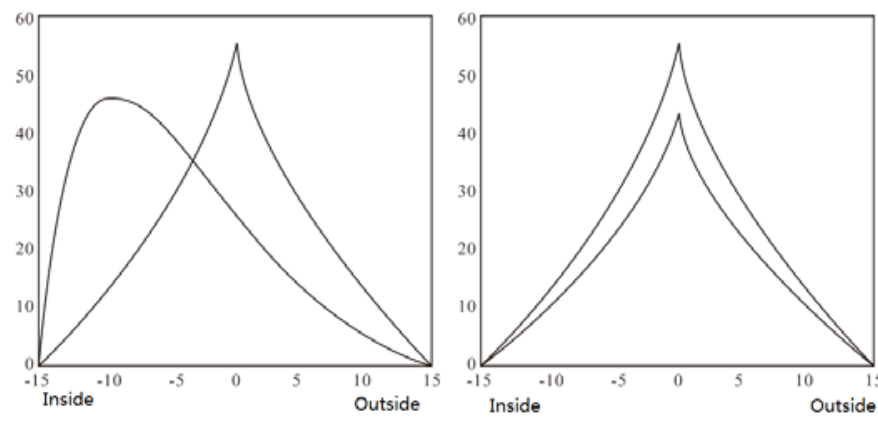

a. Before optimization

b. After optimization

Figure 2 The radius of curvature of $\mathrm{R}=60 \mathrm{~mm}$ film morphology comparison after optimization (unit: $\mathrm{mm}$ )

The simulation study confirmed that, when there is height difference or arc in the vertical direction, we can make the center axis of the gun parallel to the normal vector of the track and at the same time make sure the distance between the gun and the working surface at a constant level $h$, which makes the film morphology be the same as normal linear glue job; When there is curvature turn in plane, we can adjust the angle $\theta_{2}$ of the central axis of the spray gun and the distance $h_{y}$ between the gun and the working surface according to the radius of curvature of the corner, which can solve the uniformity problem of film thickness and width. By doing simulation experiments, we confirmed the feasibility of the optimization model.

\section{CONCLUSIONS}

Computer-controlled painting robot has been widely used in the production lines of cars, aircraft and part of the appliance, which can improve production efficiency and reduce labor costs. However, in the robot painting process, many factors can affect the quality of the painting, such as path repeat pitch, painting speed, painting pressure and painting flow. This paper dedicate to solve problem of classic film accumulation rate model and we propose a control spray control model based on paint accumulation model. Experimental results show that the improved model can solve film thickness and width uniformity issues in a better way.

\section{REFERENCE}

LIU Quanyu[1] ;TANG Zhiyong[1] ;PEI Zhongcai. Paint robot control system design based on PMAC [J].Machine Tool\& Hydraulics.2015,43(11):108-111.

HE Fujun,LI Hao.The design and ADAMS simulation of a painting mechanism used on tank wall-climbing robot[J].Manufacturing Automation,2013,35(21):107-110.

Ming Ying,Jiang Jingjue.Depth recovery from a single defocused image using a Cauchy-distribution-based point spread function model[J].Journal of Image and Graphics. 2015,20(5):708-714.

Suhs.Commuting Structure Jacobi Operator for Real Hypersurfaces Complex Two-plane Grassmannians[J].Acta Mathematica Sinica,2015,(1):111-122.

WANG Yi, ZHAO Dean, WANG Zhenbin. Comparison of two types of gun optimal trajectory planning [J].Mechanical Engineer.2014,(11):35-38.

WANG Chaojun,HE Di,PEI Xiaohui.Effects of Gauss Quadrature Schemes on the Accuracy of Full-spectrum kdistribution Method[J].Proceedings of the CSEE.2015, 35(24): 6421-6430.

XIA Wei, DU Zheng, YU Shengrui,LIAO Xiaoping. Paint spraying robot deposition rate Optimization Modeling and Simulation [J].Surface Techonology.2010,39(3):29-33.

YU Shengrui, HONG Chao, CAO Litong. Glazing thickness deposition rate modeling and forecasting of glazing robot based on artificial neural network [J]. Modular Machine Tool \& Automatic Manufacturing Technology.2011,(5):2630.

ZHAO Hongjian,ZHANG Bo.Automation automobile wheel hub painting solution[J].Manufacturing Automation. 2013, 35(14):85-87.

ZHANG Yonggui, HUANG Meiyu,GAO Feng, WANG Wei. The new model of air gun of painting robot [J]. Journal of Mechanical Engineering.2011,42(11):226-233. 